\title{
Influence of electrical phenomena on the drive train of wind power plants
}

\author{
D. Turschner ${ }^{1}$, R. Hesse ${ }^{2}$ and B. Musasa ${ }^{3}$ \\ ${ }^{13}$ Institute of Electrical Power Engineering - Clausthal University of Technology \\ Leibnizstrasse 28 \\ 38678 Clausthal-Zellerfeld \\ Germany \\ ${ }^{1}$ Phone:+0049-5323-72-2592, e-mail: dirk.turschner@tu-clausthal.de \\ ${ }^{3}$ Phone:+0049-5321-6855-125, e-mail: musasa@iee.tu-clausthal.de \\ ${ }^{2}$ IEHW Ingenieurbüro Elektrotechnik Wernigerode \\ e-mail: email@iehw.de
}

\begin{abstract}
In addition to the stress caused by the natural environment in which a wind power unit stands, it will also experience stresses due to disturbances in the electrical conditions of the power grid it is supplying.

Electrical disturbances may result from the loss of a large power consumer or producer from the power system, leading to fluctuations in voltage level and/or frequency, and the voltage may even completely collapse when one or more phases fall out or are short-circuited.

An interest of the project lies in specifying different possible load cases caused by the electrical grid in real wind power plants. A further motivation is to determine to which degree the simulation model's mechanical reaction corresponds to that of the laboratory model during grid disturbances. Wind power plants with gearbox are in the main focus of this investigation, because the damages in use are especially high here.
\end{abstract}

\section{Key words}

Wind power plant, load cases, voltage drops, fluctuation in frequency, electrical grid, short circuit, grid code

\section{Introduction}

Even though the worldwide installed wind power capacity has increased manifold over the last decade, the technology itself is still young. Unlike for instance in the auto industry, producers and developers cannot draw on a century of research and experience when designing new concepts. Therefore, prior to presenting a new wind power plant on the market, years of engineering and testing on pilot plants are needed and, because of the rapid development in the field, there is an ever present risk of the concept becoming obsolete even before it reaches the market. To speed up the development and to reduce its cost, computer simulations are naturally a very important tool in the design process. Although they can of course never give an exact picture of the physical systems they describe, simulations may offer insight into how a system reacts when it is subjected to a given set of conditions. Also, simulation models are often easier to operate than practical models and they offer an isolated environment where conditions in one part of a system can be studied without interference from its other parts.

In addition to the wear and tear caused by the natural environment in which a wind power unit (WPU) stands, it will also experience stresses due to disturbances in the electrical conditions of the power grid it is supplying. Electrical disturbances may result from the loss of a large power consumer or producer from the power system, leading to fluctuations in voltage level and/or frequency, and the voltage may even completely collapse when one or more phases fall out or are short-circuited. Induction generators (IGs), on which this paper concentrates (Fig. 1 and Fig. 2), respond especially heavily to voltage drops. Large demagnetizing currents run through the IG's stator as the voltage drops. These are reflected on the rotor and, as electromagnetic torque and rotor current are closely connected, cause strong fluctuations in the machine's torque.

In September 2003, a severe fault in the Italian distribution grid led to black-out and disconnection of the whole country from the UCTE. The UCTE frequency increased from 50.0 to 50.3 hertz in three seconds whereas it increased to 50.4 in seven seconds when a similar event occurred in Spain in December 1999. Although large voltage frequency changes are extremely rare and develop relatively slowly, their influence on a WPU must be investigated since they will certainly occur within a WPU's lifetime.

Depending on the grid operator, different standards of operation stability must be met by the power producers to be allowed onto a utility grid. The connection rules are stated in the respective operator's grid code. A grid code describes the limits for how a power generator is allowed to react to disturbances in the grid voltage, in both voltage level and frequency. Concerning IGs, the grid 
code of E.ON-Netz, a leading German operator is described in [2]. In addition to continuing supplying power as long as the grid voltage stays above a defined value, an IG WPU must support the grid by injecting a capacitive current. Also, the WPU must continue stable operation as long as the voltage frequency stays above 47.5 and below 51.5 hertz. In the following sections, a simulation model for investigating the electrical and mechanical stresses on the generator and the drive train of a WPU will be demonstrated. The model depicts a laboratory setup and is subjected to grid faults bordering the disconnection limits set by E.ON-Netz.

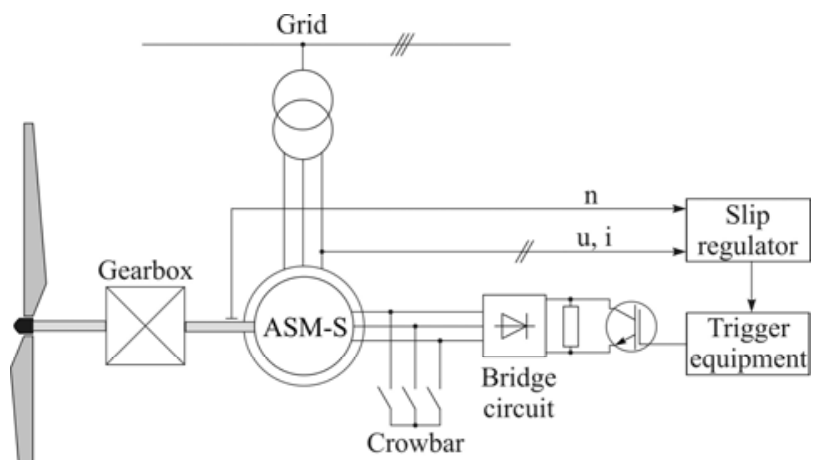

Fig. 1: Wind power plant with doubly fed generator and variable resistor in the rotor circuit.

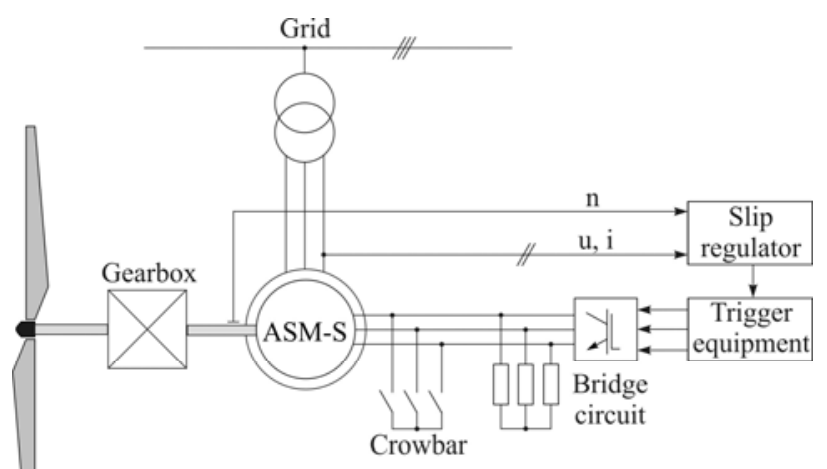

Fig. 2: Wind power plant with doubly fed generator and rotor voltage supply.

\section{Laboratory Setup}

The main motivation of this work is to determine to which degree the simulation model's mechanical reaction corresponds to that of the laboratory model during grid disturbances. The heaviest mechanical stress occurs at the mechanical resonance frequency, and the most affected part in the WPU's mechanics is the shaft linking the wind turbine to the generator due to its length and thickness.

\section{Setup Description}

From left to right the setup illustrated in Figure 4 consists of a torque-controlled $106 \mathrm{~kW}$ DC motor acting as the system's driving torque (i.e., the wind). A $200 \mathrm{~cm}$ shaft coupled to a 1:2.1 gear train transfers torque to the generator from the motor. The generator is a $30 \mathrm{~kW}$ squirrel cage IG and is connected directly to a controllable AC/AC converter imitating the power grid. A tachometer is used for measuring rotational speed and a torque sensor is used for measuring the torque applied to the shaft. For security reasons, a slipping clutch is installed. This laboratory model contains all basic parts found in a practical WPU. It is therefore argued that studying this model may give qualitative insight into a WPU's physics, despite the model's relatively small scale and small gear ratio, which is $10-100$ times greater in reality. The torque developed by DC motor is set at constant magnitude in all tests. This is reasonable since the wind driving a practical turbine will not change greatly within the short time interval studied. A photo of the test bed shows Figure 3.

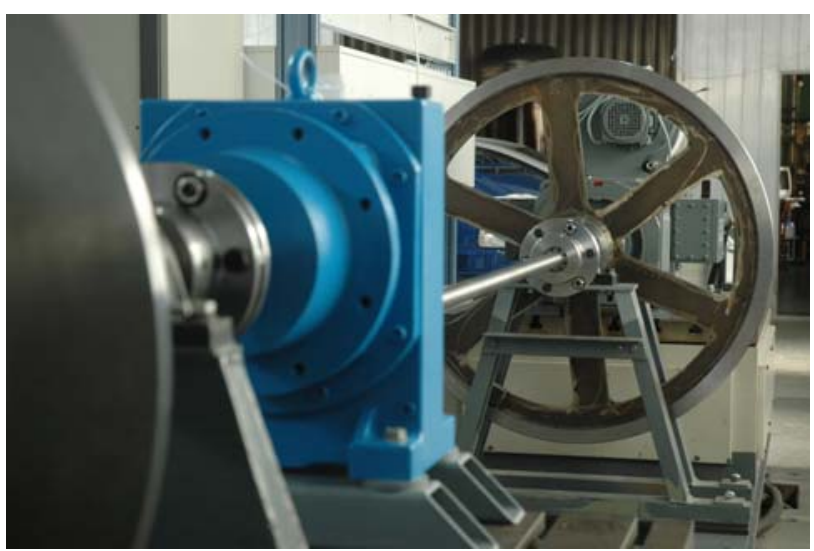

Fig. 3: Test bed with flywheel for the simulation of the turbine mass.

\section{System's Resonance Frequency}

To lower the system's resonance frequency $f_{\text {res }}$ and to strengthen the torque oscillations at $f_{\text {res }}$, a flywheel is applied at both ends of the mechanical coupling. The resulting higher inertia will also have a damping effect on the system which improves the measurements. A sufficient estimate of the resonance frequency can be made by simplifying the system. Given the low rotational stiffness of the shaft and ignoring the influence of the gear, the setup's mechanics is approximated by a linear system with two degrees of freedom. The shaft is viewed as massless and has a rotational stiffness $c_{\mathrm{T}}$. At both ends rotating discs with inertias $J_{1}$ and $J_{2}$ represent the equivalent inertias of all rotating parts left and right of the shaft in the setup. When calculating $J_{2}$, though, the gear ratio $i$ must be heeded;

$$
J_{2}^{*}=i^{2} \cdot J_{2}
$$

The simplified system's resonance frequency is calculated as follows;

$$
f_{\text {res }}=\frac{1}{2} \sqrt{c_{T}\left(\frac{1}{J_{1}}+\frac{1}{J_{2}^{*}}\right)}=6.7 \mathrm{~Hz}
$$

referred to the left and slower rotating side of the gear box. Physically measuring the resonance frequency at the shaft of the laboratory setup gives a value of about 6 
hertz, indicating that the above simplifications are reasonable.

\section{Generating Grid Faults}

Letting an AC/AC converter act as power grid, grid faults can be generated. The converter is controlled by a $D S P$ unit with state space modulation and very precisely imitates a wide range of grid disturbances such as voltage imbalances, voltage drops or collapses and frequency changes. In the case of voltage collapse imitation, the semiconductors are protected against overcurrent by firing anti-parallel thyristors connected to the generator's terminals, as illustrated in Figure 5. As mentioned above, this study is concentrated on the grid faults and a WPU must withstand without disconnecting from the power grid, according to the E.ON-Netz' grid code. Therefore, the converter is to emulate voltage drops of different magnitudes and durations and abrupt frequency changes, even though these usually develop over several seconds.

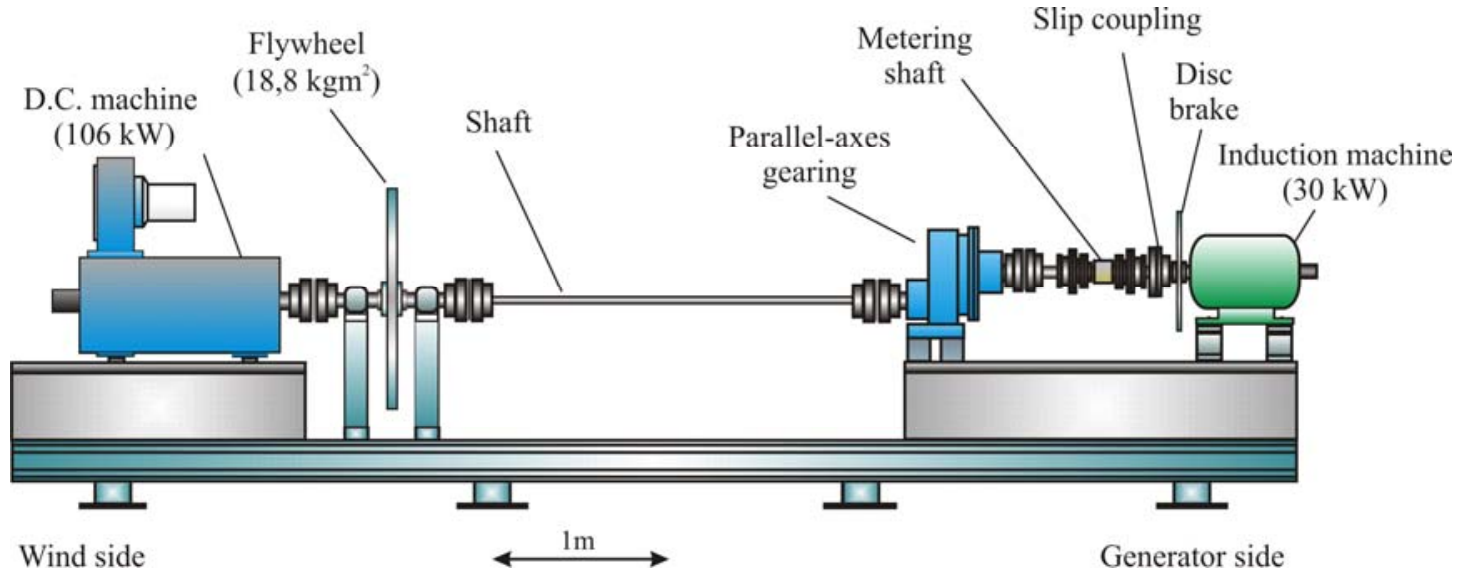

Fig. 4: General view of the test bed at the Institute of Electrical Power Engineering.

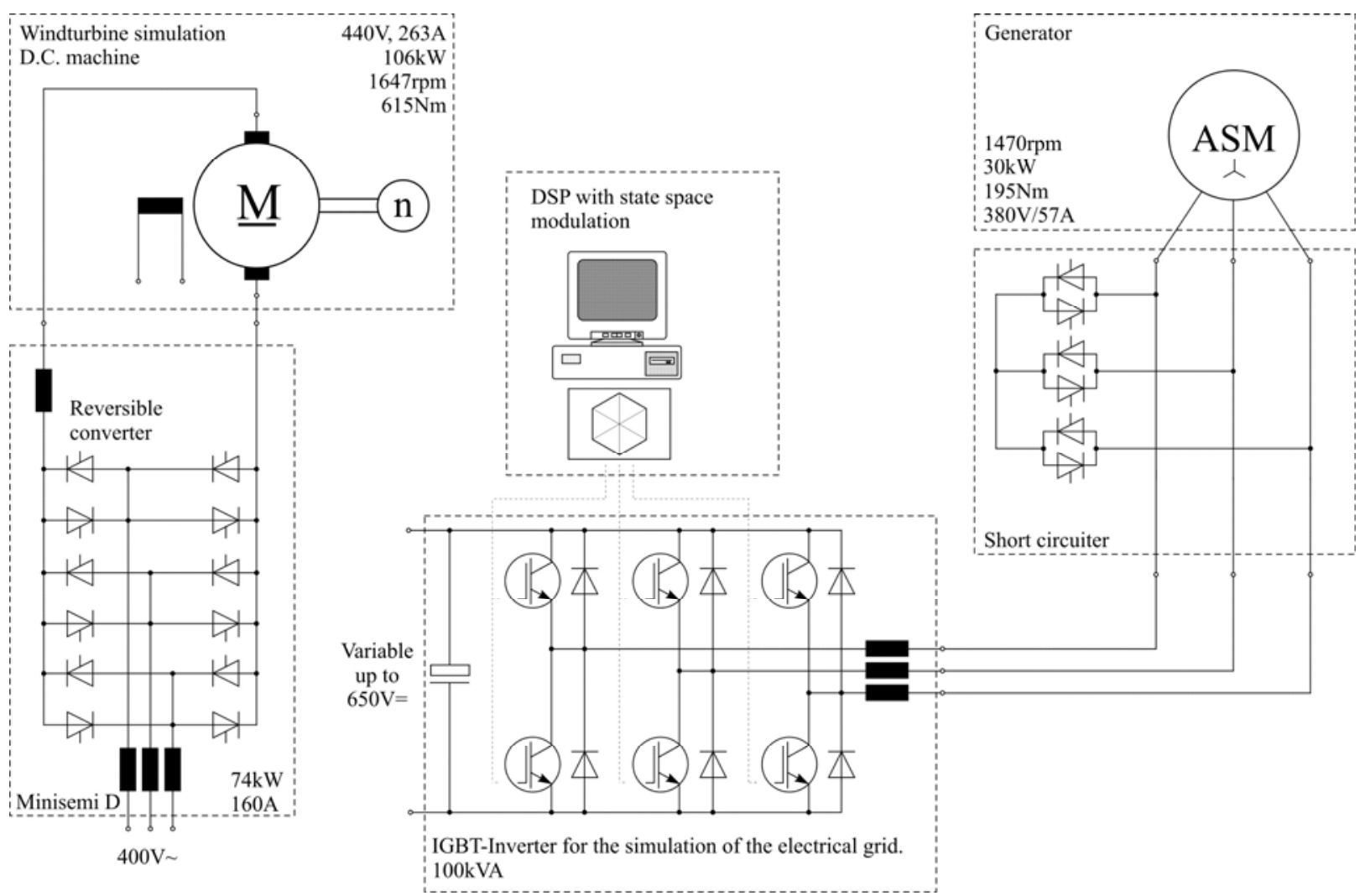

Fig. 5: Electrical description of the test bed. 


\section{Simulation Model}

Matlab Simulink provides a user-friendly graphical interface for building simulation models. The model used in this study is based on the work described in [1]. It is, as the physical model, divided into four interconnected blocks, representing the DC motor, the system's mechanics, the generator and the $\mathrm{AC} / \mathrm{AC}$ converter.

\section{Driving Torque and AC/AC Converter}

The DC motor, which in the laboratory setup is torquecontrolled, is the easiest part to model. A torque profile of any given shape is possible to create, but for the purpose of this study, a simple switch turning a constant torque on and off is sufficient. By ignoring voltage ripple, imitating the converter is also fairly straightforward. Phase-shifted sine sources represent the three phases and frequency and voltage changes are created by adjusting amplitudes and angular frequency.

\section{Mechanics}

The mechanics of the laboratory setup is described by a linear system with three degrees of freedom. The shafts can be viewed as massless when designing the simulation model. Mathematically, the system is described by the torque equilibrium equation of each of the three rotating discs. For the motor, this can be written as

$$
T_{M}=J_{M} \frac{d \omega_{M}}{d t}-T_{1}+k_{M} \omega_{M},
$$

where $k_{\mathrm{M}}$ is the frictional constant of the motor. For the generator, the equation is given as

$$
T_{G}=J_{G} \frac{d \omega_{G}}{d t}+T_{2}+k_{G} \omega_{G}
$$

The gear is driven by $T_{1}$ and braked by $T_{2}$. However, since the shafts are rotating at different speeds, the gear ratio $i$ must be included in the equation for the gear. For a frictionless gear, the following applies:

$$
i=\frac{\omega_{1}}{\omega_{2}}=\frac{T_{2}}{T_{1}}
$$

Further, if the gear as a whole, with inertia $J_{\text {Gear, }}$ is considered to be rotating at the lower (left) speed, the resulting torque equation is given as

$$
T_{1}=i T_{2}-J_{\text {Gear }} \frac{d \omega_{\text {Gear }}}{d t}-k_{\text {Gear }} \omega_{\text {Gear }}, \omega_{\text {Gear }}=\omega_{1}
$$

Here, it is important to note that $\omega_{1}$ and $\omega_{2}$ are not necessarily the same as $\omega_{\mathrm{G}}$ and $\omega_{\mathrm{M}}$ due to the two shafts' respective stiffness and damping constants. As a consequence, when calculating the parameters $T_{1}$ and $T_{2}$, $c$ and $d$ must be heeded. Doing this, the driving and braking torques are described by the following equations;

$$
\begin{aligned}
& T_{1}=c_{1}\left(\varphi_{\text {Gear }}-\varphi_{M}\right)+d_{1}\left(\dot{\varphi}_{\text {Gear }}-\dot{\varphi}_{M}\right), \\
& T_{2}=\frac{c_{2}}{2}\left[\varphi_{G}-i \varphi_{\text {Gear }}-\frac{\delta}{2} \operatorname{sign}\left(\varphi_{G}-i \varphi_{\text {Gear }}\right)\right] . \\
& {\left[1+\operatorname{sign}\left(\left|\varphi_{G}-i \varphi_{\text {Gear }}\right|-\frac{\delta}{2}\right)\right]+d_{2}\left(\dot{\varphi}_{G}-i \dot{\varphi}_{\text {Gear }}\right),} \\
& \dot{\varphi}_{M}=\omega_{M}, \dot{\varphi}_{\text {Gear }}=\omega_{\text {Gear }}, \dot{\varphi}_{G}=\omega_{G},
\end{aligned}
$$

where $\delta$ is the backlash from the gearbox and the curvedtooth Couplings of the metering shaft.

\section{Generator}

In [1], upon which this work is based, an electrical and magnetical non-linear squirrel cage machine is developed. Consequently, this simulation model does not utilize a standard machine from the Simulink library. However, the machine model is fairly complex and it is beyond the scope of this paper to go into depth on this issue.

\section{Comparison of Laboratory and Simulation Results}

The mechanical variables show very good conformance between simulation and measurement. During the 3phase-short-circuit (Fig. 6) we have at first a constant load. After a second the short-circuit is activated. A clear excessive torque appears in both directions. The system oscillates with the mechanical natural frequency. After three seconds the oscillation almost faded away.
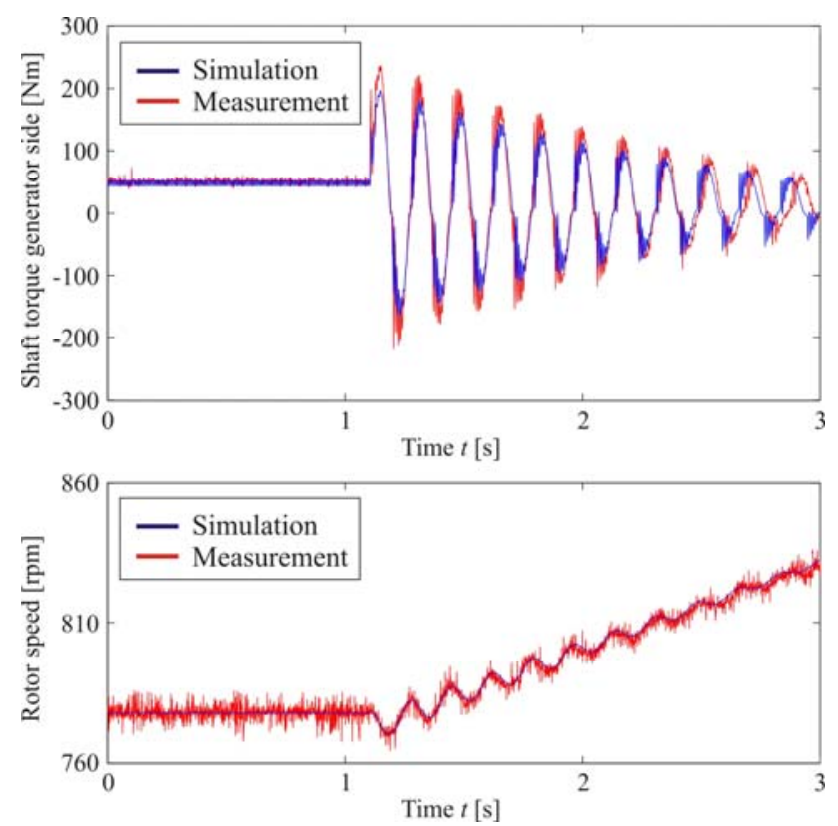

Fig. 6: Rotor speed and shaft torque of the generator side during a 3-phase-short-circuit of the stator windings.

The rotation speed goes up steeply since the active power is entered into the electrical grid. 
A frequency step from $50 \mathrm{~Hz}$ to $51.5 \mathrm{~Hz}$ shows Fig. 7 . Also here the amplitude of the shaft torque shows a clear additional stress. The rotation speed is increased.
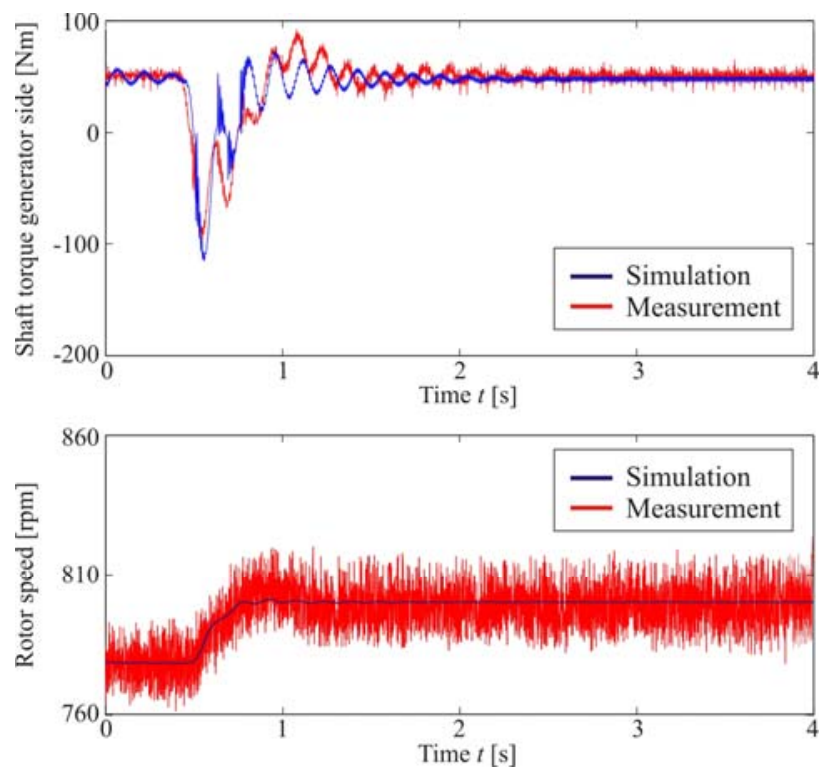

Fig.7: Rotor speed and shaft torque of the generator side during a frequency step from $50 \mathrm{~Hz}$ to $51.5 \mathrm{~Hz}$.

\section{Conclusion}

In the paper the construction of a new test bed is presented. With this equipment it is possible to simulate special load cases like 2- an 3-phase-short-circuit of the grid, reclosing of the generator in phase opposition, disconnecting of one phase, unbalanced voltage, voltage drops, frequency fluctuation, disconnecting and connecting of the generator to the grid and flicker. The results are used in order to improve the simulation. The different stress situations are needed to define special load cases. The load cases allow a gear test under real conditions.

\section{Acknowledgement}

The authors would like to thank Olav Jokob Fonstelien for the support in the case of text selection and translation into English.

\section{References}

[1] Musasa, T. B., Ein Beitrag zur Modellbildung eines elektrischen Antriebssystems mit hydrodynamischer Kupplung, Clausthal (2004), Diss.

[2] E.ON Netz GmbH, Bayreuth Netzanschlussregeln www.eonnetz.com

[3] Lubosny, Z., Wind Turbine Operation in Electric Power Systems, Advanced Modeling, Springer-Verlag Berlin 2003

[4] Turschner, D.; Hesse, R.; Beck, H.-P.;

Synchronous Machine; 9th International Conference
Electrical Power Quality and Utilisation Barcelona; 2007; 